SSN 2519-7398 (Versión electrónica)

DOI: http://dx.doi.org/10.21704/ac.v78i2.1059

(C) Universidad Nacional Agraria La Molina, Lima - Perú

\title{
Percepción de la formalidad de la cadena de reciclaje de vidrio en Lima Zona Norte
}

\author{
Perception of formality of the glass recycling chain in the North Part of Lima
}

\author{
Sonia Zamora Gonzales ${ }^{1 ;}$; Víctor Meza Contreras² \\ *Autor de correspondencia
}

\begin{abstract}
Resumen
En el Perú la cadena de reciclaje de vidrio no es ajena a la informalidad. En el presente estudio, se observó que, dependiendo de los niveles de participación, existe un menor o mayor grado de informalidad, desde los recicladores hasta las empresas fabricantes, embotelladoras y envasadoras. La presente investigación describe la percepción de la formalidad de los proveedores de la cadena de reciclaje de vidrio que inicia en la zona norte de Lima. El estudio empleó una metodología cualitativa basada en entrevistas a proveedores, especialistas y autoridades gubernamentales. La misma comprendió diferentes etapas desde la recopilación bibliográfica, el procesamiento de datos obtenidos en campo, el análisis de los resultados hasta la redacción de conclusiones y recomendaciones. Los datos se recolectaron y analizaron en el periodo de junio a noviembre del 2016. Las entrevistas de los proveedores, especialistas y autoridades gubernamentales se realizaron en las áreas de influencia de la cadena de reciclaje en la que se basa la presente investigación. Se concluye que la cadena de reciclaje de vidrio es dinámica porque no solo abarca la zona norte de Lima sino también distritos de otros conos de la ciudad. Asimismo, la percepción de formalidad varía según los niveles de trabajo y que para fomentar la formalización se sugiere realizar trabajos en conjunto con el Estado y el sector privado.
\end{abstract}

Palabras claves: percepción; formalidad; cadena de reciclaje; vidrio; zona norte de Lima.

\begin{abstract}
In Peru, glass recycling chain is familiar with informality. In this study, we have observed that, depending on participation levels, there are lower or higher levels of informality, from recyclers to manufacturing companies, bottling and packaging companies. This research study describes perceptions of formality from the standpoint of supply chain suppliers starting in the north part of Lima. The study used qualitative research methodology based on interviews with suppliers, specialists and government authorities. This study also included several stages of bibliographic collection, processing data obtained in the field, analysis of results and writing and editing conclusions and recommendations. The data was collected and analyzed in the period from June to November 2016. Interviews with suppliers, experts and government authorities were conducted in the recycling chain areas of influence on which the present research is based. It is concluded that the glass recycling chain is dynamic because it not only covers the north part of Lima but also districts of other areas of the city. As well, the perception of formality varies according to the levels of work and in order to encourage formalization, it is suggested to carry out joint work with the State and the private sector.
\end{abstract}

Key words: perception; formality; recycling chain; glass; north part of Lima.

\section{Introducción}

Según el Ministerio de Trabajo y Promoción del Empleo, en su Informe Anual del Empleo en el Perú (2014), indicó que 11.5 millones de trabajadores tienen un empleo informal, es decir, 7 de cada 10 trabajadores no cuentan con los beneficios sociales o trabajan en empresas que evaden la administración tributaria. Asimismo, la tasa de empleo informal para el Perú fue de $72.8 \%$, y para Lima y El Callao fue de 56.8\%. Loayza (2008) define al sector informal como el conjunto de empresas, trabajadores y actividades que operan fuera de los marcos legales y normativos que rigen la actividad económica. Por lo tanto, pertenecer al sector informal supone estar al margen de las cargas tributarias y normas legales, pero también implica no contar con la protección y los servicios que el Estado puede ofrecer. Las consecuencias de trabajar en un ambiente laboral informal son diversas. La Organización Internacional del Trabajo (2015) menciona algunas de ellas: menores salarios, inestabilidad laboral, precarias condiciones de trabajo, carencia de coberturas asociadas a la seguridad social y ausencia de representación y diálogo social. Por consiguiente, los trabajadores informales son un grupo vulnerable y con severas limitaciones para el desarrollo 
personal y familiar. La Ing. Albina Ruiz, Presidenta Ejecutiva de la ONG Ciudad Saludable, en una entrevista con una agencia de noticias, estimó en el 2012 que sólo el $12 \%$ de 108 mil familias recicladoras trabaja de manera formal en el Perú, por lo que se infiere que el $88 \%$ trabaja de manera informal (Andina, 2012). Las infraestructuras de almacenamiento temporal de los residuos reciclables o centros de acopio no cumplen con los requerimientos para ser formales según la Municipalidad Metropolitana de Lima (2015). Entre los principales residuos que se reciclan en el país se tiene el papel, cartón, metal, plásticos en general y vidrio. Para la presente investigación, es importante mencionar que el vidrio representa el 3.2\% de la cantidad de residuos sólidos del ámbito municipal que se genera en el Perú (Ministerio del Ambiente, 2014). Adicionalmente, el vidrio es uno de los empaques más amigables con el ambiente. De acuerdo con Owens Illinois Perú S.A. (2014), principal empresa fabricante de envases de vidrio y consumidora de vidrio reciclado en el Perú, menciona en su Informe de Sostenibilidad que el vidrio es $100 \%$ e infinitamente reciclable. Si bien existen diversos estudios sobre reciclaje de residuos municipales, muy poco se ha comentado sobre la cadena de reciclaje de vidrio, cuya actividad contribuye a la reducción de residuos que llega a diario a botaderos y rellenos sanitarios del país. No sólo tiene una importancia ambiental, sino también, el vidrio reciclado es considerado una materia prima fundamental en los procesos de fabricación de nuevos envases. Sin embargo, como toda actividad económica del país, la cadena de reciclaje de vidrio viene siendo mermada por la poca formalidad.

El objetivo principal de la presente investigación fue describir la percepción de la formalidad de la cadena de reciclaje de vidrio a nivel de proveedores de Lima Zona Norte. El estudio permite entender la forma en que los proveedores saben, piensan y sienten de su entorno. Para esto, se plantearon como objetivos específicos el identificar los flujos de la cadena de vidrio reciclado, analizar las diferentes percepciones de formalidad de los proveedores de la cadena de reciclaje y proponer alternativas de mejora de la cadena de reciclaje de vidrio a nivel de proveedores de Lima Zona Norte.

\section{Materiales y métodos}

La investigación es de carácter cualitativo dado que la percepción es un factor subjetivo. Se diseñó un cuestionario que permitió recabar información a través de entrevistas abiertas, con lo cual se buscó indagar los puntos de vista de los entrevistados, utilizando medios como la conversación, observación e interpretación de los datos producto de las entrevistas. Las entrevistas abiertas forman parte del método de investigación cualitativo. De acuerdo con Hernández et al. (2010), el enfoque cualitativo busca comprender la percepción de los participantes acerca de los fenómenos que los rodean, profundizar en sus experiencias, perspectivas, opiniones $\mathrm{y}$ significados, es decir, la forma en que los participantes perciben subjetivamente su realidad. Las entrevistas se realizaron en la ciudad de Lima y Callao entre los meses de junio y septiembre del 2016. Se entrevistó un total de doce personas divididas en cuatro grupos: proveedores de vidrio reciclado de Lima Zona Norte, proveedores de vidrio reciclado de otras zonas de Lima, especialistas en el tema de logística inversa y autoridades gubernamentales relacionadas al rubro de residuos sólidos. Las personas entrevistadas fueron seleccionadas por conveniencia.

\section{Diseño de la investigación}

El diseño para una investigación de enfoque cualitativo es el narrativo, pues se recolectan datos sobre las experiencias y puntos de vistas de expertos sobre un tema en particular para luego describirlos y analizarlos (Hernández et al., 2010). La investigación contó con los siguientes pasos generales: (i) Recolección de datos; (ii) Revisión de la información recabada y; (iii) Sistematización e interpretación de los resultados.

\section{Recolección de datos}

La recolección de datos se realizó a través de entrevistas abiertas. Los proveedores entrevistados de la cadena de vidrio reciclado, tanto de la zona norte y otras zonas de Lima, son los mayores abastecedores de materia prima de Owens Illinois Perú y Envases de Vidrio - Envisac, principales empresas fabricantes de envases de vidrio en Lima Metropolitana. Las entrevistas se realizaron con el apoyo de una grabadora de voz, así como, de fotografías y apuntes. Asimismo, se contó con un cuestionario o formato de entrevista (Anexo 1), la cual incluye las preguntas que se realizaron vinculadas con el planteamiento del problema y objetivos de investigación. Hernández et al (2010) indica que las entrevistas abiertas se fundamentan en una guía general de contenido y el entrevistador posee toda la flexibilidad para manejarla, de esta forma los participantes expresan de la mejor manera sus experiencias y sin ser influidos por la perspectiva del investigador. Posterior a las entrevistas realizadas a los proveedores de vidrio reciclado, se desarrollaron entrevistas complementarias. Este segundo grupo estuvo conformado por autoridades gubernamentales vinculados al área de residuos sólidos y especialistas en logística inversa. La finalidad de entrevistar a este grupo de actores fue de entender el distanciamiento que hay entre la percepción de los proveedores de la cadena de reciclaje de vidrio versus la percepción de autoridades y especialistas. Las entrevistas se realizaron con el apoyo de una grabadora de voz y se contó con un cuestionario o formato de entrevista (Anexo 2).

Los entrevistados fueron categorizados y codificados con finalidad de mantener la confidencialidad de los mismos.

\section{Revisión de la información recabada}

En esta segunda etapa, se procedió a analizar el material y la información recabada por categoría y codificación mediante la transcripción de las entrevistas. Hernández et 
al (2010) indica que se debe transcribir las grabaciones de audio y video producto de las entrevistas, a fin de hacer un análisis exhaustivo del lenguaje del entrevistado. Asimismo, durante la revisión del material, se detectó que algunas entrevistas contaban con información parcial, por lo que se procedió a realizar una entrevista adicional. El objetivo de la revisión es asegurar que el material este completo y posea la calidad necesaria para ser analizado (Hernández et al., 2010).

\section{Sistematización e interpretación de los resultados}

La tercera etapa consistió en sistematizar la información de forma cualitativa y cuantitativa, tomando como base la propuesta de Aguilar et al. (2011). El análisis cualitativo se realizó mediante un cruce de información en una matriz de doble entrada en el que se resume la entrevista realizada por categorías (proveedores, autoridades y especialistas). El análisis cuantitativo consistió en elaborar una Tabla de tabulación de datos por categorías y de manera global, tanto en porcentaje como en proporción.

\section{Muestra}

El tipo de muestreo es intencional cuando los sujetos de la muestra no son elegidos siguiendo las leyes de azar ni siguiendo un cálculo o ley de probabilidades, sino de alguna forma conveniente (Aguilar et al., 2011). Adicionalmente, Hernández et al. (2010) sugiere el muestreo por conveniencia cuando se tiene casos disponibles a los cuales uno tiene acceso, es decir, el investigador decide que individuos pasan a formar parte de la muestra en función a la disponibilidad de los mismos. Aguilar et al. (2011) indica que, dentro del muestreo cualitativo, una de las modalidades principales es el muestreo opinático, en donde el investigador selecciona los informantes que han de componer la muestra siguiendo un criterio estratégico personal: los más fáciles, los que voluntariamente o fortuitamente son los más idóneos y representativos de la población a estudiar, o entran en contacto con el investigador a través de sujetos entrevistados previamente. Para la presente investigación, el tipo de muestreo fue intencional opinático. El número de personas consideradas fue de doce, divididas de la siguiente manera.

Para la selección de la muestra se consideró los siguientes criterios estratégicos:

- Proveedores que formen parte de la cadena de abastecimiento de vidrio reciclado de Owens Illinois Perú y Envases de Vidrio - Envisac, principales empresas fabricantes de envases de vidrio en Lima Metropolitana.

- Proveedores de vidrio reciclado cuya área de trabajo se enfoque, principalmente, en la zona norte de Lima. Esta zona está conformada por los siguientes distritos: Ancón, Santa Rosa, Puente Piedra, Carabayllo, Comas, Los Olivos, Independencia y San Martín de Porres. Asimismo, se consideró adicionar para la investigación, los distritos de Ventanilla y Callao, debido a que, representan un área importante en el flujo de materiales reciclables ya que se encuentra cercano al Relleno Sanitario Modelo del Callao.

- Especialistas o profesionales que tengan relación con logística inversa.

- Autoridades del sector público que tengan experiencia en el desarrollo o ejecución de programas de manejo de residuos sólidos.

Tabla 1. Determinación de la muestra de estudio

\begin{tabular}{cc}
\hline Categoría & $\mathrm{N}^{\circ}$ de personas \\
\hline Proveedores de Lima Zona Norte & 05 \\
Proveedores de otras zonas de Lima & 02 \\
Especialistas en logística inversa & 03 \\
Autoridades gubernamentales & 02 \\
Total & 12 \\
\hline
\end{tabular}

\section{Confidencialidad}

Con la finalidad de mantener en reserva los nombres de las personas entrevistadas, por el principio de confidencialidad, se procedió a categorizar y codificar a los entrevistados. Las categorías fueron divididas en proveedores de Lima Zona Norte, proveedores de otras zonas de Lima, especialistas en logística inversa y autoridades gubernamentales. Luego de separar a los entrevistados en categorías, se procedió a asignarles una codificación, tal como se muestra en la Tabla 2 .

Tabla 2. Codificación de las categorías de entrevistados

\begin{tabular}{cc}
\hline Codificación & Descripción \\
\hline PZnN & Proveedor de Lima Zona Norte \\
POZn & Proveedor de otras zonas \\
ELogI & Especialista en logística inversa \\
AGb & Autoridad Gubernamental \\
\hline
\end{tabular}

\section{Resultados y Discusión}

\section{Descripción de la cadena de reciclaje de vidrio de Lima Zona Norte}

De acuerdo con lo observado en campo y la información recabada en las entrevistas realizadas, la cadena de reciclaje de vidrio de la zona norte de Lima se divide en dos flujos. El primer flujo es el mercado de comercialización de vidrio roto, el cual tiene como destino final las empresas fabricantes de nuevos envases. El segundo flujo es el mercado de reuso, en especial de envases de licores, el cual no ha sido objeto de investigación del presente estudio. Sin embargo, se puede mencionar que la merma de este mercado, producto de la selección de envases, forma parte de la cadena de abastecimiento del primer flujo.

Se puede indicar que el mercado de comercialización de vidrio roto en Lima Zona Norte presenta cuatro niveles de participación: recolectores/generadores, acopiadores, proveedores y fabricantes, tal como se observa en la Figura 1. 


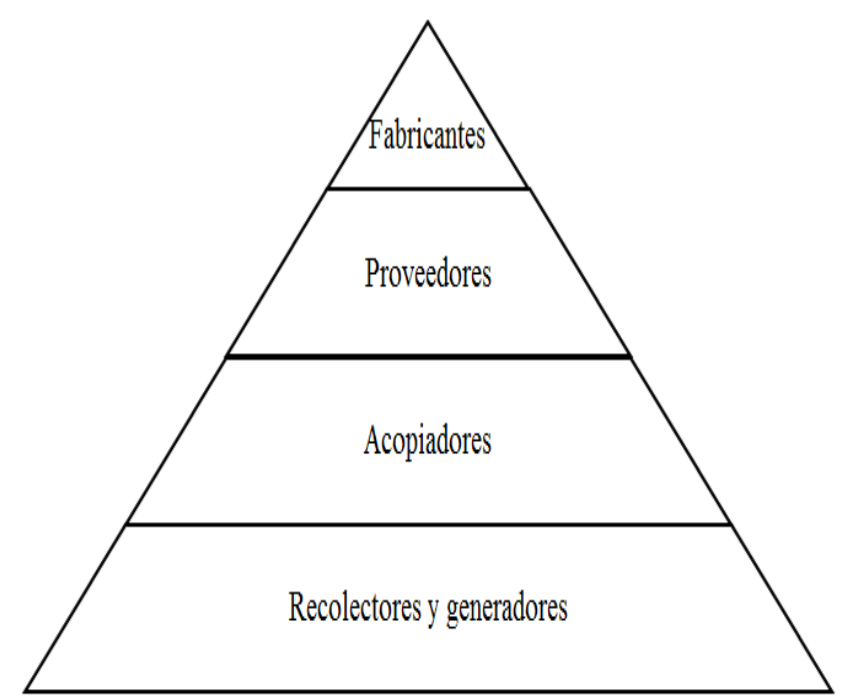

Figura 1. Pirámide de la cadena de reciclaje de vidrio de Lima Zona Norte

El primer nivel o base de la cadena está conformado por los recolectores y generadores. Los recolectores son aquellas personas que realizan las labores de recolección de los residuos reciclables en la fuente de origen. Conforman este grupo, los recicladores y el personal del área de limpieza pública de la Municipalidad. Tomando como base el estudio de Ciudad Saludable (2010) y la población estimada por el INEI (2015), se presenta en la Tabla 3, la cantidad estimada de recicladores y de centros de acopio en Lima Zona Norte. Se puede observar que el número de recicladores de la zona norte de Lima y de los distritos de Ventanilla y Callao es de 16,534 individuos. De acuerdo con Ciudad Saludable (2010), un reciclador puede recolectar entre 26 a $500 \mathrm{~kg}$ en promedio al mes de residuos reciclables, ganando entre 400 a 1,050 soles. Por otro lado, los generadores están conformados por personas, familias o pequeñas empresas que generan, en su día a día, residuos reciclables. Tanto los recolectores como los generadores, trasladan los residuos reciclables a los centros de acopio para su venta. La comercialización del vidrio reciclado se realiza bajo dos formas: venta de envases de vidrio por peso y venta de envases de licores por unidad. Durante la transacción, no se genera ningún tipo de documento contable y el pago se realiza al contado.

El segundo nivel está conformado por acopiadores que cuentan con locales o centros de acopio donde se realiza la compra y venta de los residuos reciclables. De acuerdo con la Tabla 3, la cantidad estimada de acopiadores de Lima Zona Norte es de 2,828 individuos. De acuerdo con Ciudad Saludable (2010), un acopiador puede comercializar entre 1 a 15 toneladas al mes con un capital de trabajo mínimo de unos 2,000 soles mensuales. Conforme a lo observado en campo, la cantidad promedio de acopio de vidrio reciclado varía desde $200 \mathrm{~kg}$ hasta $4,000 \mathrm{~kg}$ al mes. A este nivel de la cadena, el vidrio comprado es seleccionado y separado para venta como vidrio entero o preparado (roto), y para el mercado de reuso (licor). La venta de vidrio roto consiste en que los acopiadores trituran los envases para luego comercializarlo por colores (blanco, ámbar y verde). En cambio, la venta de vidrio entero consiste en acumular en sacos, los envases íntegros sin separarlos por color. Son los proveedores, pertenecientes al nivel posterior, los que se encargan de preparar el vidrio. Por otro lado, tal como menciona Ciudad Saludable (2010), los acopiadores operan de manera formal e informal. Son pocos los centros de acopio que entregan un comprobante de pago. De acuerdo con los proveedores entrevistados, los recicladores y acopiadores consideran que pueden obtener una mayor ganancia evitando el pago de los impuestos y el contrato de un contador.

Tabla 3. Cantidad estimada de recicladores en Lima Zona Norte

\begin{tabular}{lrrr}
\hline \multicolumn{1}{c}{ Distritos } & Población & Recicladores & Acopiadores \\
\hline Ancón & 39,600 & 198 & 34 \\
Carabayllo & 301,978 & 1,510 & 258 \\
Comas & 524,894 & 2,624 & 449 \\
Independencia & 216,822 & 1,084 & 185 \\
Los Olivos & 371,229 & 1,856 & 317 \\
Puente Piedra & 353,489 & 1,767 & 302 \\
San Martin de Porres & 700,178 & 3,501 & 599 \\
Santa Rosa & 18,751 & 94 & 16 \\
Callao & 406,889 & 2,034 & 348 \\
Ventanilla & 372,899 & 1,864 & 319 \\
\hline Total & $3,306,729$ & 16,534 & 2,828 \\
\hline
\end{tabular}

Los proveedores integran el tercer nivel de la cadena. Son empresas constituidas legalmente, conforme a lo indicado por Ciudad Saludable (2010), y principales abastecedores de vidrio reciclado de las empresas fabricantes de envases de vidrio, entre ellas, la transnacional Owens Illinois y la empresa Envases de Vidrio - Envisac. Se caracterizan por cubrir más de dos distritos de la zona norte de Lima. Sin embargo, cabe indicar que sus centros de trabajos no necesariamente se ubican en la zona referida, sino que se encuentran en otros distritos como El Callao y Ventanilla, distritos donde existe un flujo considerable de materiales reciclables ya que se encuentra cercano al Relleno Sanitario Modelo del Callao, en cuyos alrededores se localizan una gran cantidad de centros de acopio. A través de la presente investigación, se pudo identificar a siete proveedores de vidrio reciclado de Lima, de los cuales sólo cinco abarcan la zona norte de Lima. De acuerdo con la mayoría de los proveedores entrevistados, la cantidad de vidrio reciclado que mueven al mes varía entre 26 a 350 toneladas, con un capital promedio de 5,200 a 80,000 soles. La comercialización del vidrio roto que mantienen con las empresas fabricantes se realiza mediante la emisión de facturas y guías de remisión.

El cuarto nivel lo conforma las empresas fabricantes de envases de vidrio. Tal como menciona Ciudad Saludable (2010), la principal demandante de vidrio reciclado 
en el Perú es la empresa Owens Illinois, la cual tiene como principal línea de producción, la fabricación de envases para alimentos, bebidas no alcohólicas y bebidas espirituosas. Asimismo, se puede mencionar a Envases de Vidrio - Envisac como otra empresa referente en la cadena de reciclaje de vidrio ya que es consumidora de vidrio blanco (flint) y ámbar reciclado para la fabricación de diversos tipos de recipientes de vidrio. Por otro lado, existen empresas demandantes, en su mayoría, de vidrio ámbar, Ecoglass Perú, Cristalería Langard y Amfa Vitrium, compañías que se dedican a cubrir las necesidades de las industrias farmacéuticas.

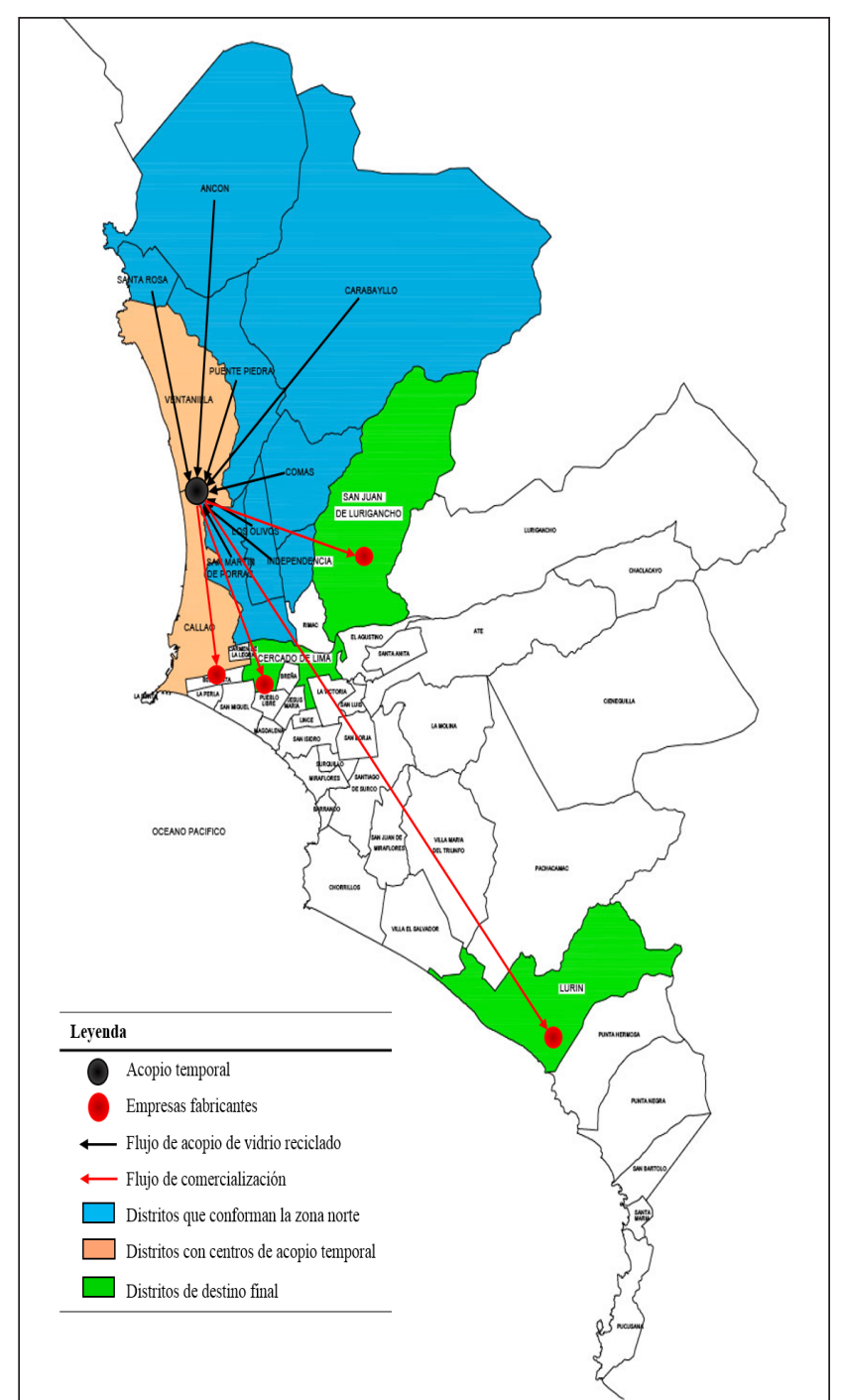

Figura 2. Ruta de reciclaje de vidrio de la zona norte de Lima

Adicionalmente, se puede mencionar que el flujo de la cadena de vidrio reciclado de Lima Zona Norte es bastante dinámico ya que traspasa diferentes distritos. Tal como se observa en la Figura 2, si bien la cadena inicia con la recolección del vidrio en la zona norte, el acopio temporal se realiza en almacenes de los proveedores ubicados principalmente en El Callao o Ventanilla, o van directamente a los almacenes o plantas de fabricación de envases de vidrio. La empresa Owens Illinois cuenta con dos plantas ubicadas en los distritos de Bellavista y Lurín. De acuerdo con Ciudad Saludable (2010), la cantidad de vidrio reciclado que demanda Owens Illinois es de cerca de 30,000 toneladas al año entre vidrio blanco, verde y ámbar. Las plantas de fabricación de las empresas Envases de Vidrio - Envisac, Ecoglass y Cristalería Langard se ubican en el distrito de San Juan de Lurigancho, y Amfa Vitrium en el Cercado de Lima.

\section{Análisis de la percepción de la formalidad de la cadena de reciclaje de vidrio de Lima Zona Norte}

\section{Análisis de los resultados de las entrevistas a proveedores}

De acuerdo con los resultados de las entrevistas realizadas a los proveedores de la cadena de reciclaje de vidrio $\mathrm{y}$ tomando como base las 25 preguntas formuladas $\mathrm{y}$ descritas en el Anexo 1, se puede mencionar los siguientes resultados generales:

- El vidrio reciclado es sostenible e imperecedero. De acuerdo con los proveedores, el vidrio es un material que no se deteriora, no se malogra y se conserva en el tiempo. Tal como se menciona en la revisión bibliográfica, Campodónico et al. (2011) afirma que el vidrio es un material que se caracteriza por su dureza, elevada resistencia química y es $100 \%$ e infinitamente reciclable.

- El vidrio reciclado es considerado como un material de dificultosa maniobrabilidad. Para los proveedores de la Zona Norte, el vidrio reciclado representa un material peligroso, pesado y trabajoso.

- El mercado de vidrio reciclado, en especial el vidrio blanco, es regulada principalmente por una empresa. De acuerdo con los proveedores entrevistados y Ciudad Saludable (2010), la empresa de mayor demanda de vidrio reciclado en el Perú es la transnacional Owens Illinois. Se podría inferir que la cadena de vidrio reciclado en Lima es un mercado con características de monopolio.

- La mayoría de los proveedores que forman parte de la cadena de reciclaje de vidrio son micro y pequeñas empresas (MYPE) que acopian en promedio entre 1 a 15 toneladas diarias, enmarcadas dentro del D.S. $\mathrm{N}^{\circ}$ 057-2004/PCM (2004). Asimismo, tomando en cuenta el precio promedio de compra de Owens Illinois, la cadena de reciclaje de vidrio está conformada por micro y pequeñas empresas, según lo estipulado en el D.S. N013-2013-PRODUCE (2013).

- Los proveedores de vidrio reciclado no cumplen, en su totalidad, con el marco normativo relacionado a la promoción de micro y pequeñas empresas ya que sólo el $57.1 \%$ de los entrevistados manifestaron que cumplen con la jornada de ocho horas diarias de trabajo, enmarcadas en el D.L. $\mathrm{N}^{\circ} 1086$. El 42.9\% restante superan dicho horario. Asimismo, el D.L. $\mathrm{N}^{\circ} 1086$ señala que los trabajadores tienen derecho 
de contar con un SCTR, un seguro médico (SIS o ESSALUD) y a un sistema de pensiones (ONP o AFP). Sin embargo, sólo el $57.1 \%$ de los proveedores entrevistados cumplen con brindar un SCTR y sólo dos proveedores de Lima Zona Norte manifestaron que cuentan con personal en planilla que gozan con seguro médico y fondo de pensiones.

- El precio base de compra de vidrio reciclado se ha mantenido constante en el tiempo. El importe de compra de los centros de acopio se ha sostenido en 10 céntimos de sol por kilo desde hace más de dos décadas. De acuerdo con Ciudad Saludable (2010), el reciclador o base de la cadena percibe por la venta de vidrio reciclado entre 5 a 10 céntimos de sol por kilo.

- La cadena de reciclaje de vidrio está orientada a ser formal porque la comercialización principalmente se sostiene con la empresa Owens Illinois. De acuerdo a lo mencionado por los proveedores entrevistados, la mayoría maneja su negocio con dicha empresa quien les exige contar con RUC, guías de remisión y facturas para ser considerados como proveedores de su cadena. Sin embargo, en niveles por debajo de los proveedores, es decir, a nivel de centros de acopio y recicladores de pie, la cadena de vidrio reciclado se caracteriza por un flujo constante de dinero cuyas transacciones se realizan con pago a contra entrega sin la emisión de un documento contable, por lo que se infiere que la cadena, a este nivel, está orientada a la informalidad.

- Los proveedores de la cadena de vidrio reciclado no cumplen con las disposiciones legales ambientales ya que no cuentan con el registro de EC-RS/EPS-RS, conforme a lo estipulado en la Ley No27314 (2000). Asimismo, el D.S. $\mathrm{N}^{\circ} 005-2010-\mathrm{MINAM} \mathrm{(2010)}$ señala que las personas que se dedican al reciclaje deben contar con equipos de protección personal
(EPP). De acuerdo con las entrevistas realizadas, sólo $71.4 \%$ de los proveedores señalaron que cumplen con tal disposición. Sin embargo, conforme a las observaciones en campo y al testimonio de algunos de los proveedores, los trabajadores no necesariamente emplean los EPP en campo.

- Por otro lado, la NTS N073-MINSA/DIGESA (2008) menciona que las personas que laboran en reciclaje deben contar con examen médico pre-ocupacional y de control periódico, pero ninguno de los proveedores entrevistados cumple con lo mencionado.

- Se percibió que los proveedores de la cadena de reciclaje de Lima Zona Norte no tienen claro el concepto y beneficios que pueden obtener si se registran como EC-RS/EPS-RS ya que los resultados no muestran un patrón en común y relacionan el concepto directamente con DIGESA.

- Con la finalidad de agrupar las respuestas más relevantes relacionadas a temas de formalidad, se muestra la Figura 3, una relación de cuatro categorías divididas de la siguiente manera:

- Empresa constituida: si el proveedor cuenta con RUC, emite guías de remisión y genera facturas.

- Conocimiento teórico: si el proveedor conoce y sabe de las funciones de la SUNAT y DIGESA.

- Cumplimiento legal ambiental: si el proveedor cuenta con el registro como EC-RS / EPS-RS, cumple con los EPP necesarios para desarrollar la actividad de reciclaje, y si lleva a cabo los exámenes médicos correspondientes por Ley.

- Cumplimiento legal empresarial: si el proveedor cuenta con personal en planilla, cumple con el horario de trabajo, y brinda seguro médico, fondo de pensiones y SCTR.

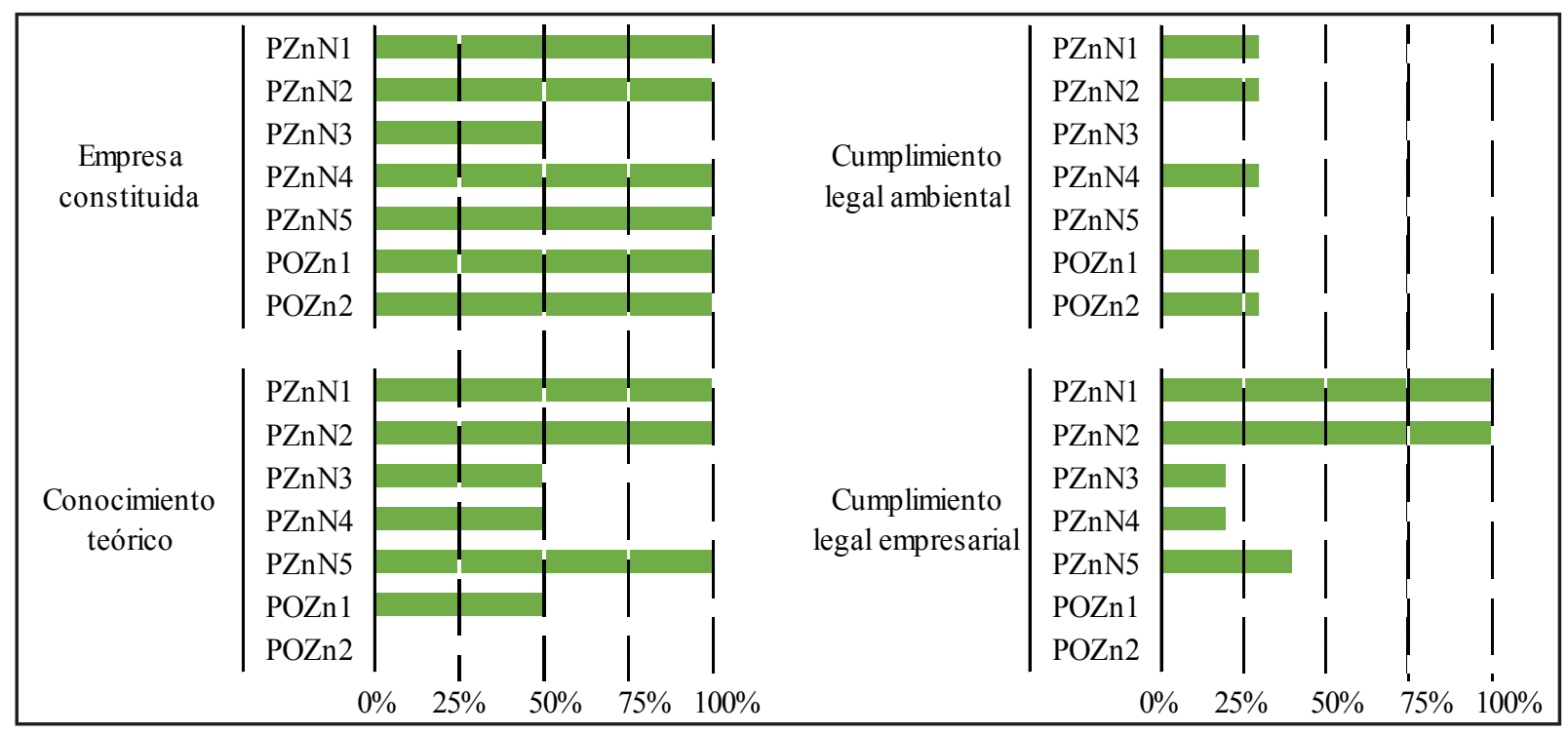

Figura 3. Aspectos que enmarcan la formalidad de la cadena de reciclaje de vidrio 
En general, se puede mencionar que, si bien la mayoría de los proveedores son empresas constituidas que conocen medianamente sobre las funciones de la SUNAT y beneficios de estar registrados ante la DIGESA, no cumplen al 100\% con las disposiciones legales ambientales ni empresariales solicitadas para ejercer el reciclaje de vidrio.

Con relación a los proveedores de la cadena de reciclaje de vidrio de la zona norte de Lima, se puede indicar que cuatro de cinco entrevistados se encuentran constituidos como empresa, de los cuales solo tres de ellos tienen presente el concepto de SUNAT y DIGESA. Asimismo, en cuanto al cumplimiento legal ambiental, se observa que sólo tres proveedores de la zona norte cumplen con un poco más del $25 \%$ de los requerimientos. Por último, con relación al cumplimiento legal empresarial, sólo dos proveedores cumplen al $100 \%$ con los requisitos de planilla, horario de trabajo, seguro médico, fondo de pensiones y SCTR.

\section{Análisis de los resultados de las entrevistas a proveedores}

Luego del análisis de las 05 preguntas realizadas a los especialistas y autoridades gubernamentales de la cadena de reciclaje de vidrio, cuyo formato de entrevista se encuentra en el Anexo 2, se puede mencionar los siguientes resultados generales:

- La cadena de vidrio reciclado es considerada, por los especialistas y autoridades gubernamentales, como un flujo con características de poca formalidad.

- Los costos y las demoras en la gestión de los trámites ante DIGESA o entidades gubernamentales son considerados una traba en el proceso de formalización de la cadena de reciclaje de vidrio. Tanto los especialistas como las autoridades gubernamentales entrevistados, manifestaron que las diligencias pueden ser muy engorrosas, extensas y a la vez caras que no permiten avanzar en los planes de formalización de los negocios.

- Existe un flujo de recuperación de envases de vidrio entre dos grandes empresas: la fabricante Owens Illinois y la embotelladora SABMiller.

- La cadena de reciclaje de vidrio cuenta con diferentes oportunidades de mejora relacionadas principalmente a la creación de alianzas entre el sector público y privado.

\section{Alternativas de mejora de la cadena de reciclaje de vidrio a nivel de proveedores de Lima Zona Norte}

Para los proveedores entrevistados, una de las mejoras que se debería dar es la conciliación de un precio de compra fijo. Asimismo, la mejora en infraestructura y equipos de los locales, la obtención del registro como EC-RS/EPS-RS, el fomento de programas educacionales para la población y programas de capacitación para los proveedores.

Por otro lado, los especialistas y autoridades gubernamentales consideraron que las mejoras de la cadena residen principalmente en formar alianzas entre El Estado y el sector privado, a fin de fomentar políticas que conlleven a impulsar el reciclaje. De esta forma, lograr que la cadena de reciclaje este de la mano con el cumplimiento de los estándares ambientales, administrativos y operativos que subraya la legislación peruana.

En general, se puede mencionar las siguientes acciones como alternativas de mejora de la cadena de reciclaje de vidrio a nivel de proveedores:

- Creación de un programa de asistencia técnica relacionados a temas de reciclaje y formación empresarial impulsado por el Estado, a través del Ministerio de Ambiente, y en alianza con el sector privado tomando como base las características de los proveedores de la cadena.

- Desarrollar talleres participativos en los que se impulse la importancia de tener un negocio formal a través del apoyo de especialistas del Ministerio de Trabajo y Promoción del Empleo, así como, de la SUNAT.

- Fomentar la obtención del registro de EC-RS/EPS-RS a través de canales de información mediante el cual el proveedor pueda ser asesorado por especialistas de DIGESA a fin de que puedan cumplir con los requerimientos necesarios.

- Brindar asesoramiento a los proveedores para el acceso a créditos bancarios de baja tasa de interés mediante el apoyo de representantes de entidades financieras a fin de que puedan invertir en la habilitación de sus locales y en la adquisición de equipos y vehículos.

\section{Conclusiones}

El flujo de reciclaje de vidrio de Lima Zona Norte es dinámico ya que existe una relación constante entre los cuatro niveles de la cadena (recolectores/generadores, acopiadores, proveedores y fabricantes), así como, abarca distritos de otros conos de la ciudad, como la zona sur donde se ubica la empresa Owens Illinois o el cono este donde se ubican las empresas Envases de Vidrio, Ecoglass y Cristalería Langard. Asimismo, la cadena inicia con una base estimada de 16,534 recicladores y 2,828 acopiadores. Asimismo, se identificaron siete proveedores de vidrio reciclado las cuales abastecen a las empresas fabricantes, que se ubican en el tope de la pirámide, conformadas por Owens Illinois, Envases de Perú, Ecoglass, Cristalería Langard y Amfa Vitrium. Por otro lado, el 85.7\% de los proveedores entrevistados son empresas constituidas que emiten tanto guías de remisión como facturas, y conocen moderadamente sobre las funciones de la SUNAT y los beneficios de obtener el registro de EC-RS o EPS-RS ante DIGESA. Sin embargo, no cumplen al 100\% con las disposiciones legales ambientales ni empresariales solicitadas para ejercer el reciclaje de vidrio. Con relación a la percepción de formalidad de la cadena de vidrio reciclado de los proveedores, se puede afirmar que varía según los niveles de trabajo. Se pudo percibir que la percepción de los proveedores a niveles inferiores de la cadena es de un flujo informal. Sin embargo, la percepción que tienen ellos hacia niveles superiores de la cadena 
es formal por el hecho de trabajar directamente con las empresas fabricantes de envases de vidrio. Asimismo, la cadena de vidrio reciclado es un mercado con potencial de crecimiento en temas de formalidad ya que existe una gran empresa que viene regulando el precio y solicitando el cumplimiento de ciertos requisitos legales y documentarios. Sin embargo, a nivel de centros de acopio y recicladores de pie, aún falta mucho por trabajar para ello se requiere de un trabajo exhaustivo y coordinado entre el sector privado, el Estado y la sociedad. Finalmente, las propuestas de mejora de la cadena de reciclaje de vidrio a nivel de proveedores están encaminadas a desarrollar acciones que busquen fomentar su formalización en su totalidad, a través de trabajos en conjunto con el Estado, como una entidad de asistencia técnica y especialista en el tema, y con el sector privado, como parte interesada en obtener mayores niveles de acopio.

\section{Literatura citada}

Andina. 2012. Agencia Peruana de Noticias. Sólo el $12 \%$ de recicladores trabaja de manera formal en el Perú, informan. Lima, PE. Consultado abr. 2016. Disponible en: http://www.andina.com.pe/agencia/ noticia-solo-12-recicladores-trabaja-manera-formalel-peru-informan-396247.aspx

Aguilar, C. et al. 2011. Percepción de la comunidad universitaria sobre la implementación de programas de ecoeficiencia. Tesis MBAG. Lima, PE. Centro de Negocios de la Pontificia Universidad Católica del Perú. 143 p.

Campodónico, G. et al. 2011. Planeamiento estratégico de la industria del vidrio. Tesis MBAG. Lima, PE. Centro de Negocios de la Pontificia Universidad Católica del Perú. 143 p.

Ciudad Saludable. 2010. Por la ruta del reciclaje en el Perú: Estudio socioeconómico de la cadena del reciclaje. 1 ed. Lima, PE. 350 p.

Decreto Legislativo $N^{\circ}$ 1086. 2008. Ley de Promoción de la Competitividad, Formalización y Desarrollo de la Micro y Pequeña Empresa y del Acceso al Empleo Decente. Lima, PE.

Decreto Supremo No 057-2004-PCM. 2004. Reglamento de la Ley General de Residuos Sólidos. Lima, PE.

Decreto Supremo No 005-2010-MINAM. 2010. Reglamento de la Ley que Regula la Actividad de los Recicladores. Lima, PE.

Decreto Supremo No 013-2013-PRODUCE. 2013. Texto Único Ordenado de la Ley de Impulso al Desarrollo Productivo y al Crecimiento Empresarial. Lima, PE.

Hernández, R. et al. 2010. Metodología de la investigación. 5 ed. México D.F., MX. McGraw-Hill Interamericana Editores. 613 p.

INEI (Instituto Nacional de Estadística e Informática). 2015. Censo poblacional. Lima, PE. Consultado nov. 2016. Disponible en http://proyectos.inei.gob.pe/web/ poblacion/

Ley $N^{\circ}$ 27314. 2000. Ley General de Residuos Sólidos. Lima, PE.

Loayza, N. 2008. Causas y consecuencias de la informalidad en el Perú. Banco de Reserva del Perú. Lima, PE. Revista Estudios Económicos no. 15:43-64.

MML - Municipalidad Metropolitana de Lima. 2015. Diagnóstico ambiental de la provincia de Lima. Lima, PE. 154 p.

MTPE - Ministerio de Trabajo y Promoción del Empleo. 2015. Informe anual del empleo en el Perú 2014. Lima, PE. 124 p.

Norma técnica $\mathrm{N}^{\circ}$ 073-2008-MINSA/DIGESA. 2008. Norma Técnica de Salud que Guía el Manejo Selectivo de Residuos Sólidos por Segregadores. Lima, PE.

Owens - Illinois. 2014. The most sustainable package on Earth: Sustainability Report. Ohio, US. 60 p.

OIT - Organización Internacional del Trabajo. 2015. Formalizando la informalidad juvenil: experiencias innovadoras en América Latina y el Caribe. 1 ed. Lima, PE. Oficina Regional para América Latina y el Caribe. $71 \mathrm{p}$. 


\section{ANEXOS}

\section{Anexo 1: Guía de entrevista a proveedores}

Fecha: Hora:

Lugar (dirección, distrito):

Entrevistadora:

Entrevistado(a) (nombre, rango de edad, cargo):

\section{Introducción}

Descripción general de la investigación (propósito, motivo por el cual fueron elegidos, utilización de datos).

Buenos (dias/tardes). Mi nombre es Sonia Zamora. Soy estudiante de la Universidad Nacional Agraria La Molina. La presente entrevista forma parte de la investigación "Percepción de la formalidad de la cadena de reciclaje de vidrio en Lima Zona Norte" y tiene como propósito la obtención del Título de Ingeniera Ambiental. Los resultados de la investigación son para fines académicos.

Siendo las (horas) del (fecha) del (mes) del 2016, procedo a entrevistar al (Sr./Sra)(nombre del entrevistado) que forma parte de la cadena de reciclaje de (Owens Illinois Perú/Envases de Vidrio - Envisac).

\section{Preguntas}

1. ¿Desde cuándo se dedica al reciclaje?

2. ¿Cómo inició su negocio de reciclaje?

3. Desde que empezó su negocio, ¿cómo ha cambiado el reciclaje?

4. ¿Cómo ve Usted el negocio de reciclaje en el futuro? (cuáles son sus proyecciones)

5. ¿Qué tipo de materiales recicla?

6. Sabiendo que Usted pertenece a la cadena de reciclaje de (O-I/Envisac) ¿Qué tipo de vidrio comercializa?

7. ¿Qué opina del mercado de reciclaje de vidrio?

8. ¿Cómo acopia el vidrio? (quiénes le venden, donde lo almacena)

9. ¿Qué cantidad de vidrio recicla al mes? (por tipo/ rango de cantidades)

10. ¿Realiza algún tratamiento al vidrio? (lavado, triturado, ensacado)

11. ¿Cuenta con RUC?

12. ¿Cuenta con guías de remisión y facturas?

13. ¿Siempre emite guías y facturas cuándo comercializa vidrio?

14. ¿Cómo es la forma de pago hacia sus proveedores y de sus clientes? (plazo y frecuencia de pago, modo de pago)

15. ¿Cómo es su relación con sus colaboradores? (personal)

16. ¿Reciben algún incentivo o beneficio por trabajar con
Usted? (seguro médico, EPP, planilla)

17. ¿Su local es propio?

18. ¿Cuenta con licencia de funcionamiento?

19. ¿Ha contado con la visita de la Municipalidad?

20. ¿Qué documentos le solicita la Municipalidad para poder laborar?

21. ¿Alguna vez trató de tramitar su registro de EC-RS o EPS-RS? (se explicará al entrevistado(a) el significado de EC-RS o EPS-RS)

22. ¿Cuenta con permiso del INDECI o Defensa Civil?

23. ¿Está al día en el pago de sus impuestos?

24. ¿Qué opina/qué sabe de la SUNAT?

25. ¿Qué cree que se necesita para mejorar la cadena de reciclaje de vidrio?

Anexo 2: Guía de entrevista a especialistas en logística inversa y autoridades gubernamentales

Fecha: Hora:

Lugar (dirección, distrito):

Entrevistadora:

Entrevistado(a) (nombre, rango de edad, cargo):

\section{Introducción}

Descripción general de la investigación (propósito, motivo por el cual fueron elegidos, utilización de datos).

Buenos (dias/tardes). Mi nombre es Sonia Zamora. Soy estudiante de la Universidad Nacional Agraria La Molina. La presente entrevista forma parte de la investigación "Percepción de la formalidad de la cadena de reciclaje de vidrio en Lima Zona Norte" y tiene como propósito la obtención del Título de Ingeniera Ambiental. Los resultados de la investigación son para fines académicos.

Siendo las (horas) del (fecha) del (mes) del 2016, procedo a entrevistar al (Sr./Sra)(nombre del entrevistado) que se desempeña como (especialista/autoridad).

1. ¿Qué opina de la cadena de reciclaje en el Perú?

2. ¿Cómo describirías la cadena de logística inversa de la empresa donde usted labora actualmente?

3. ¿Qué opinas de los recicladores y cuál crees que es su papel que juegan dentro de la cadena de reciclaje de vidrio?

4. ¿Cuál crees que son las oportunidades que existen para mejorar la cadena de reciclaje?

5. ¿Cuál consideras que es papel del (Sector Privado/ Estado) dentro de la cadena de reciclaje? 